\title{
The glucose tolerance test in mice: sex, drugs and protocol.
}

\author{
Matilda Kennard ${ }^{1}$, Manasi Nandi², Sarah Chapple ${ }^{1}$, and Aileen King ${ }^{1}$ \\ ${ }^{1}$ Kings College London \\ ${ }^{2}$ King's College London
}

July 1, 2021

\begin{abstract}
Background and Purpose: Glucose tolerance tests (GTTs) are commonly used in preclinical studies but are poorly standardised. Male mice are often preferred due to more severe phenotypes which may aid in detecting drug effects. Using novel glucose telemetry in undisturbed mice, the effect of different pre-GTT protocols on blood glucose concentrations, GTTs and detection of drug effects were considered. Experimental Approach: Seven male and female C57Bl/6J mice (8-10 weeks) were implanted with HD-XG glucose telemetry devices. Mice were fasted for $16 \mathrm{~h}$ overnight or $6 \mathrm{~h}$ in the daytime following a whole cage change, cage change with retention of used bedding or no cage change prior to i.p.GTTs. Glucose tolerance following oral glucose gavage was compared to voluntary ingestion of gels. Using the most refined procedures, $250 \mathrm{mg} / \mathrm{kg}$ oral metformin and $10 \mathrm{nmol} / \mathrm{kg}$ i.p. exendin-4 were tested. Key Results: Blood glucose initially increased following cage changing at the start of the fast. For $6 \mathrm{~h}$ fasting, retaining bedding reduced these initial responses and produced more timely glucose reductions whereas $16 \mathrm{~h}$ fasts caused pronounced hypoglycaemia. Impaired glucose tolerance in males was exaggerated following $16 \mathrm{~h}$ fasting or whole cage changes. Refined procedures including voluntarily ingested glucose gels blunted responses but the effects of exendin- 4 and metformin were still observable in both sexes. Conclusion and Implications: Variations in GTT protocol can have profound effects on glucose homeostasis. Improved glucose tolerance due to protocol refinement and/or the use of females still allows for detection of drug effects providing evidence that more severe phenotypes are not required when testing drugs.
\end{abstract}

The glucose tolerance test in mice: sex, drugs and protocol.

Running title: Glucose tolerance test in mice.

Kennard, M.R. ${ }^{1}$, Nandi, M. ${ }^{2}$, Chapple, S. $^{3}$, King, A.J.F. ${ }^{1}$

${ }^{1}$ Department of Diabetes, King's College London, London, UK ${ }^{2}$ Institute of Pharmaceutical Science, King's College London, London, UK ${ }^{3}$ School of Cardiovascular Medicine \& Sciences, King's College London, London, UK

Word count: 3932

Acknowledgements: This work was funded by the British Pharmacological Society through an AJ Clark studentship awarded to MRK. We also gratefully acknowledge the BSU staff at KCL for the excellent care and advice regarding the research animals.

Conflict of interest: The authors declare no conflicts of interest.

Abbreviations: GTT: glucose tolerance test CGM: continuous glucose monitoring P-E: proestrous-estrous M-D: metestrous-diestrous WCC: whole cage change BRCC: bedding retention cage change NCC: no cage change

What is already known: 
Glucose tolerance tests (GTTs) are poorly standardised despite differing protocols having potential impacts on both welfare and scientific outcomes.

Females are largely excluded from preclinical diabetes research due to perceived variability due to the estrous cycle and attenuated diabetic phenotype.

\section{What this study adds:}

Small variations in protocols can have profound effects on blood glucose concentrations affecting welfare, efficacy of fasting and GTT outcome.

Proof that animals with lower blood glucose concentrations, whether due to protocol refinement or sex, are suitable to observe drug effects.

\section{Clinical significance}

Improvement of welfare whilst maintaining predictive validity can optimise animal models for drug discovery.

Inclusion of females, which is recommended in this study, could improve translatability of drug discovery.

\section{Introduction}

Animal models play an important role in preclinical diabetes research with glucose tolerance tests (GTTs) being a popular tool for detecting impaired glucose homeostasis (Andrikopoulos et al., 2008; McGuinness et al., 2009). GTTs are also often used to test new anti-diabetic treatments as the efficacy of blood glucose lowering drugs is easier to determine when blood glucose has been acutely increased via a bolus of exogenous glucose (Pacini et al., 2013). Hence GTTs are one of the most commonly undertaken experiments in preclinical diabetes research.

Female mice are often excluded from diabetes research, as they become less glucose intolerant and insulin resistant following induction of the disease in almost all models (Kaikaew et al., 2019; Pettersson et al., 2012). Indeed, this relative lack of phenotype is commonly believed to impede their usefulness in drug efficacy studies. Females are also often excluded due to perceived increased variability in blood glucose concentrations across the estrous cycle. This perception is grounded by known effects of estrogen on blood glucose homeostasis, including enhancing glucose tolerance and reducing insulin resistance (Mauvais-Jarvis, 2015). However, to the best of our knowledge, the impact of sex and the estrous cycle on blood glucose variability has not previously been studied in detail, despite growing emphasis on the need to consider sex as a biological variable in preclinical studies (Beery, 2018; Clayton \& Collins, 2014; Docherty et al., 2019).

Despite their importance, GTTs are poorly standardised in preclinical settings, with lab-to-lab variation in almost all aspects of the protocol. Fasting is undertaken prior to GTTs to reduce the influence of variability in food intake and ensure that blood glucose concentration is primarily being maintained by liver homeostasis. Despite overnight fasting (corresponding to 15-18h) previously being very common, it has been associated with significant welfare implications including weight loss (7-16\%), hypoglycaemia, hypothermia and several cardiovascular changes (Jensen et al., 2013). Consequently, shorter fast lengths, primarily in the daytime, are now preferred with evidence that $4-6 \mathrm{~h}$ fasts are sufficient to reduce blood glucose concentration, ensure gastric emptying and increase hepatic glucose production (HGP) (Ayala et al., 2010; Champy et al., 2004; Heijboer et al., 2005; Prior et al., 2012). Despite this, a literature search using the key phrase 'glucose tolerance and mouse' revealed that $60 \%$ of studies in the last 3 years have still undertaken overnight fasts prior to GTTs.

As fasting is undertaken to eliminate the influence of food, some researchers may change their mouse's cages at the start of the fast to ensure that no food remnants are present at the bottom. However, research has shown that whole cage changes in $\mathrm{C} 57 \mathrm{Bl} / 6 \mathrm{~J}$ mice are associated with various stress responses including increased corticosterone, increased blood pressure and anxiety-like behaviours (Rasmussen et al., 2011). This is most likely due to mice relying heavily on their olfactory systems with cages being scented with many familiar smells which are essential in maintaining the mouse's society and hierarchy (Rasmussen et 
al., 2011). Consequently cage changes, where used bedding is retained, has been shown to reduce stress responses associated with whole cage changes (Rasmussen et al., 2011).

The act of administering glucose could also affect GTT outcome beyond that of well-established differences in routes of administration due to the incretin effect (Seino \& Yabe, 2013). In most cases, animal restraint is required for glucose administration by either intraperitoneal (I.P.) injection or oral gavage which could lead to stress responses and subsequent changes in blood glucose homeostasis (Turner et al., 2011). However, mice can be trained relatively easily to consume a glucose gel which allows unrestrained glucose administration and therefore would be regarded as further refinement. Indeed, oral gels have been shown to be effective in both the context of GTTs (Cox et al., 2010; Zhang, 2021) and drug delivery (Dhawan et al., 2018; Zhang, 2011). However, there are potential limitations of this method including the need for training, the possibility of non-adherence and the need for mouse separation which could impact welfare (Pedro et al., 2020; Zhang, 2021).

Whilst previous research has addressed the lack of GTT standardisation in diabetes research, there is a lack of comprehensive data showing the effect of different experimental and physiological variables chosen by the researcher (Andrikopoulos et al., 2008; Ayala et al., 2010). This is essential to not only tackle biases in experimental design (such as excluding females) but to also highlight to researchers that seemingly innocuous parts of the protocol could be affecting outcome. One major hurdle is that in order to measure blood glucose concentrations, researcher intervention is normally required. Continuous glucose monitoring (CGM) is now available in unrestrained mice requiring surgical implantation of an indwelling glucose monitoring probe. CGM allows for comprehensive and continuous analysis of blood glucose concentrations with minimal disturbance to the animal. This has enabled us to consider the real-time impact of various interventions on blood glucose concentration and to determine whether any changes in blood glucose can be minimised via refinement. Furthermore, understanding the impact of refinement, sex and estrous cycling is not just important for improving welfare and translatability of research, but is essential for ensuring predictive validity of the model. Indeed, it is vital that any refined procedures still support the generation of reproducible and scientifically valid data such as allowing for drug effects to be detected. Consequently, we sought to determine whether refined procedures and the inclusion of female mice altered the ability to detect clinically relevant drug effects during GTTs.

\section{Methods}

\section{Animals}

Seven male and eight female C57Bl/6J mice (8-10 weeks and 20-30g) (Charles River, UK) were implanted with HD-XG glucose telemetry probes (Data Science International, USA) after two weeks of acclimatisation and baseline measurements of weight and blood glucose concentration via glucometer (StatStrip Xpress, NovaBiomedical, USA). Furthermore, nine non-telemetered male and nine non-telemetered female C57Bl/6J mice of the same age and weights were also studied in separate experiments.

All experiments were approved by the institution's welfare and ethics committee and undertaken in accordance with the UK Animals (Scientific Procedures) Act 1986 with 2012 amendments. C57Bl/6J mice were chosen due to their frequent use in diabetes research both when normoglycaemic and as a background strain for diabetic models (Jensen et al., 2016; King, 2012).

All mice were housed in a controlled environment at $22^{\circ} \mathrm{C}$ with free access to standard Rodent Diet 20 chow (Picolab, UK) and water with 7am-7pm light:dark cycles. Mice were housed in open cages with nonlittermate buddies of the same sex which, for telemetry cages, comprised of one telemetered mouse and one non-surgical buddy. Appropriate enrichment including cardboard houses and tunnels was placed in each cage. All experimentation was undertaken in specialised telemetry holding rooms without the need to move the mice. At the end of the study all mice were killed via intraperitoneal overdose of anaesthetic $(2 \mathrm{~g} / \mathrm{kg}$ Euthatal; Merial Animal Health, UK) with secondary cervical dislocation following dissection of telemetry probes. 
Pilot glucose tolerance test (GTT) experiments were undertaken in non-telemetry male mice to determine sample sizes for telemetry experiments. The difference in means $(3.2 \mathrm{mM})$ and expected standard deviation $(1.9 \mathrm{mM})$ for the 15 -minute time-point of a $6 \mathrm{~h}$ fasted intraperitoneal GTT following a whole cage change vs bedding retention cage change was used. Criteria was set at a power of 0.80 and alpha of 0.05 and calculations revealed $n=7$ was required to see differences. Consequently, we implanted probes into 8 male and 8 female telemetry mice to allow for potential surgical complications.

Mice were randomised for glucose probe telemetry surgery with probes randomised to each mouse. Telemetry probe fasting data and glucose tolerance test experiments were undertaken with $n=7$ for both males and females. A further experiment considering the effect of gavage vs gel glucose tolerance was undertaken in 5 non-telemetry male and female mice $\mathrm{C} 57 \mathrm{Bl} / 6 \mathrm{~J}$ mice (8-10 weeks) and drug testing experiments used $\mathrm{n}=4$ due to this being a proof-of-concept study primarily undertaken for sample size calculations.

\section{Surgical implantation of glucose telemetry probes and recovery}

Anaesthesia was induced in animals with $2.5-5 \%$ isoflurane in 1 litre/minute $95 \%$ oxygen and maintained throughout surgery with $1.5-2 \%$ in 0.5 litre/minute oxygen via a facemask. Mice were maintained at $37^{\circ} \mathrm{C}$ via a homeothermic heating blanket and rectal probe. Mice were administered $4 \mathrm{mg} / \mathrm{kg}$ carprofen (Carprieve; Centaur, UK) in sterile saline (AquaPharm; Centaur, UK) subcutaneously prior to probe implantation and $2 \mathrm{mg} / \mathrm{kg}$ Marcain (Centaur, UK) at the wound site immediately prior to surgical recovery. A further $4 \mathrm{mg} / \mathrm{kg}$ dose of carprofen was administered $24 \mathrm{~h}$ post-surgery. The sensor portion of the probe was advanced towards the aortic arch, accessed via the left carotid artery, and sutured in place whilst the radio-transmitter portion was advanced subcutaneously under the right abdominal flank along with $\sim 0.5 \mathrm{ml}$ saline resuscitation. Animals were recovered on their own in incubators overnight at $28^{\circ} \mathrm{C}$ after which they were transferred to the monitoring lab and reintroduced to their buddies.

Eight male and female mice were implanted with telemetry probes. Animals were closely observed for seven days whereby blood glucose concentration (by tail tip glucometer), weights and clinical observations (surgical wound site, behaviour, feeding patterns) were monitored. All mice were fed a combination of solid and mashed standard chow diet for the first three days post-surgery then transferred to a normal solid chow diet only. Mice progressively lost weight in the first $48 \mathrm{~h}$ post-surgery with maximum losses of $1.7 \pm 0.2 \mathrm{~g}(5.8 \pm 0.8 \%)$ for males and $1.5 \pm 0.2 \mathrm{~g}(6.4 \pm 1.0 \%)$ for females on day two. By day five, body weight had stabilised in both sexes. One male mouse was culled on day 1 post-surgery due to suspected stroke and one female mouse was culled on day 2 post-surgery due to ill health with an unknown cause. This resulted in $n=7$ for both groups.

\section{Probe calibration}

An initial multi-point probe calibration occurred on day three post-surgery. Calibration was undertaken using a StatStrip Xpress blood glucose meter and strips (Novabiomedical, USA) with blood samples obtained from the tail vein using a $30 \mathrm{G}$ needle. Following a baseline measurement, blood glucose was increased using $2 \mathrm{~g} / \mathrm{kg}$ intraperitoneal injection of glucose (Sigma-Aldrich, UK) in sterile saline (AquaPharm; Centaur, UK). Single-point calibrations were then undertaken three times weekly for the duration of the study with a final multi-point calibration undertaken at the end of experimentation in line with manufacturer's instructions.

\section{Estrous swabbing and staining}

Vaginal smears were obtained for 10 consecutive days to determine estrous cycling in each female mouse. Smears were then obtained at the end of each glucose tolerance test and immediately prior to refeeding. Smears were placed on to slides and stained with methylene blue for 12 minutes (Sigma-Aldrich, UK). Mice were staged based on the relative proportions of nucleated epithelium, cornified epithelium and leukocytes as observed with a light microscope (Byers et al., 2012). Mice were separated into a proestrous-estrous (P-E) group, when estrogen is normally high, and a metestrous-diestrous (M-D) group, when estrogen is normally low.

Effect of cage change method and fast length on blood glucose concentration 
Telemetered mice were fasted for $6 \mathrm{~h}$ during the daylight cycle (from 9am-3pm) with a whole cage change, bedding retention cage change or no cage change at the start of the fast (Table 1). Mice were also fasted for $16 \mathrm{~h}$ (commencing at $5 \mathrm{pm}$ ) with a bedding retention cage change only.

Whole cage changing (WCC) consisted of the mice being handled via the tail and placed in a new cage with new wood chippings, new bedding and new enrichment. Bedding retention cage changing (BRCC) consisted of mice being handled by the tail and placed in a new cage with new wood chippings but the bedding and enrichment from the old cage was retained and placed in the new cage. No cage changing (NCC) consisted of food being removed from the cage lid only. Mice were also fasted for $16 \mathrm{~h}$ overnight (commencing at $5 \mathrm{pm}$ ) with a bedding retention cage change (BRCC) at the start of fasting (Table 1).

\section{Effect of cage change method and fast length on glucose tolerance test outcome}

At the end of $6 \mathrm{~h}$ or $16 \mathrm{~h}$ fasting, mice were weighed and a baseline blood glucose concentration was measured via glucometer (Novabiomedical, UK). Mice were then administered $2 \mathrm{~g} / \mathrm{kg}$ glucose (Sigma-Aldrich, UK) in sterile saline (AquaPharm; Centaur, UK) via intraperitoneal injection. Repeat blood glucose concentrations were then measured discontinuously at 15-, 30-, 60-, 90- and 120-min following glucose administration, from the tail vein using a glucometer. Telemetry probes simultaneously measured blood glucose concentrations every 10 seconds for the entire 120 min duration of the GTT.

\section{Effect of voluntary oral route of glucose administration}

Based on results from the above, refined procedures were used to determine whether further refinement of oral glucose administration was possible using voluntarily consumed gels.

$60 \%$ oral glucose gels were made using $80 \%$ (w/v) D-glucose (Sigma-Aldrich, UK) in distilled water. This was mixed with gelatine (Dr Oetker) in water with sugar-free chocolate flavouring in a 3:1 dilution. Mice weights from $24 \mathrm{~h}$ prior to the GTT were used to make glucose gels to administer a dose of $2 \mathrm{~g} / \mathrm{kg}$. Mice were separated from their buddies whilst gels were administered using wooden cage separators.

Mice were trained to eat the glucose gels over one week. Initially, mice were given the gels on two consecutive days with ad libitum food. Mice were then acclimatised to the cage separators by separating them from their buddy for $2 \mathrm{~h}$. Following this, mice were fasted for $6 \mathrm{~h}$ (9am-3pm daylight cycle) with a bedding retention cage change before being separated for 30 minutes prior to administration of the glucose gel on two separate occasions. Mice were considered trained when they consistently ate the gel within 1 minute. Gels were weighed in their pots before administration with any uneaten remnants weighed after administration. Time taken to eat the gel was also measured. Gels were removed after 60 seconds and the mice were reunited. Any mouse that ate less than $90 \%$ of the gel within this 60 second period was excluded from analysis.

The final optimised protocol was selected and consequently mice were fasted for $6 \mathrm{~h}$ during the daylight cycle $(9 \mathrm{am}-3 \mathrm{pm})$ with a bedding retention cage change. At the end of the fast, a baseline blood glucose concentration was obtained before administration of $2 \mathrm{~g} / \mathrm{kg}$ glucose (Sigma-Aldrich, UK) in sterile saline (AquaPharm; Centaur, UK) via oral gavage or $2 \mathrm{~g} / \mathrm{kg}$ glucose (Sigma-Aldrich, UK) in distilled water using oral gels. Repeat discontinuous blood glucose concentrations were then measured at 15-, 30-, 60-, 90- and 120min using a glucometer. Telemetry probes simultaneously measured continuous blood glucose concentrations every 10 seconds for the entire duration of the GTT.

\section{Effect of drugs using refined procedures}

Based on the data generated, the procedures that combined the most refined method with least variable fasting data was identified as a $6 \mathrm{~h}$ fast commencing at 9am following a bedding retention cage change (BRCC). Using these refined procedures, we next wished to assess the impact of known blood glucose lowering pharmacological interventions and whether their effects are measurable and reproducible. 30 minutes prior to the end of the fast, a baseline blood glucose concentration was obtained simultaneously via glucometer and telemetry. Mice were then administered either a) $250 \mathrm{mg} / \mathrm{kg}$ metformin (Sigma-Aldrich, UK) via oral gels, using sugar-free sucralose sweetener as control, or b) $10 \mathrm{nmol} / \mathrm{kg}$ exendin-4 (Sigma-Aldrich, UK) via 
intraperitoneal (I.P.) injection using saline as control to mimic the clinically relevant routes of administration. 30 minutes after drug or control administration, $2 \mathrm{~g} / \mathrm{kg}$ glucose was administered via voluntary ingestion of glucose gels with repeat discontinuous blood glucose concentrations measured at 15-, 30-, 60-, 90- and 120min as before. Telemetry probes also obtained continuous blood glucose concentrations every 10 seconds for the duration of the GTT.

Metformin gels were made using $0.18 \%$ (w/v) 1,1-Dimethylbiguanide hydrochloride (Sigma-Aldrich, UK) in distilled water. This was mixed with gelatine (Dr Oetker) in water and sucralose sweetener (Splenda) in water with chocolate flavouring (Nick's Stevia drops) in a 1:1:1 dilution. Sweetener control gels were made by mixing sucralose sweetener in water with chocolate flavouring and gelatine in water in a 2:1 dilution. As with glucose gels, mice weights from $24 \mathrm{~h}$ prior to the GTT were used to make both metformin and glucose gels. Mice were separated using cage separators for the duration of both metformin and glucose gel administration with any remnants of gel being weighed to determine volume ingested.

\section{Data and statistical analysis}

The data and statistical analysis comply with the recommendations on experimental design and analysis in pharmacology (Curtis et al., 2018).

Mice were separated into three cohorts: males, females in proestrous-estrous (P-E) and females in metestrousdiestrous (M-D) for all experiments apart from the drug glucose tolerance tests due to the preceding results. As swabs were obtained after experimentation, this resulted in $n=7$ for males, $n=5-7$ for females in P-E and $\mathrm{n}=4-7$ for females in M-D. Continuous glucose time-series data was acquired using Ponemah software v6.x (Data Sciences International, USA) with 10 second window averages exported as Excel files for analysis.

To consider the effect of fasting and cage change method, 10 second averages of glucose time series data were analysed from 30 minutes prior to the start of the fast until immediately prior to the start of the GTT. Initial plotting of data showed a biphasic response to fasting and cage changing with an initial increase in blood glucose in response to human intervention (0-120 minutes) and then a secondary decrease in blood glucose due to the fast (120 minutes to end of fast).

The initial increase in glucose was consequently quantified using $\mathrm{AUC}_{0-120}$ (overall response), the difference between pre-intervention and maximum blood glucose (magnitude of increase) and the percentage time spent above pre-intervention glucose in this timeframe (duration of increase). The pre-intervention blood glucose concentration was calculated as the average blood glucose from -30 to -15 minutes prior to any intervention for each mouse, ensuring that results were not affected by the researcher entering the room.

The secondary decrease in glucose from 120-330 minutes for 6h fasting and 120-900 minutes for 16h fasting was then analysed by calculating the minimum blood glucose concentration (magnitude of reduction) and percentage time below pre-intervention glucose within this timeframe (duration of reduction). Results were compared to normal $24 \mathrm{~h}$ values under ad libitum conditions for the same mice. The time at which blood glucose concentration first fell below pre-intervention levels for at least 5 consecutive minutes was also analysed. All data for both the initial and secondary phases were normally distributed with equal variance between groups and statistically analysed using two-way ANOVA with Holm-Sidak post-hoc tests.

Glucose tolerance test data was analysed for each of the three cohorts following the different fast lengths and/or cage change methods. Blood glucose concentrations from 0-120 minutes were normally distributed with equal variance between groups and statistically analysed using two-way RM ANOVA with Holm-Sidak post-hoc tests for each cohort. $\mathrm{AUC}_{120}$ was also calculated for each mouse and compared between both fast length/ cage change method and cohort using two-way RM ANOVA with Holm-Sidak post-hoc tests. The same was true for gavage vs gel GTTs and drug vs control GTTs although $\mathrm{AUC}_{150}$ was calculated for drug vs control due to the -30 minute pre-drug baseline.

To ascertain whether different numbers of male and female mice were required for testing drugs, sample size calculations were undertaken for drug vs control GTTs using the difference in means and standard deviations 
between $\mathrm{AUC}_{150}$ for both drugs vs their respective controls and the 15 minute post-glucose blood glucose concentrations. Criteria was set at a power of 0.90 and alpha of 0.01 .

All graphical and statistical analysis was undertaken on SigmaPlot 14.0, including the quantification of AUCs and sample size calculations. All data is presented as mean+-standard error of the mean (SEM) and p $<0.05$ was taken to represent a significant difference.

\section{Materials}

Euthatal was obtained from Merial Animal Health, UK. Carprofen (Carprieve), sterile saline (AquaPharm) and Marcain were obtained from Centaur, UK. D-Glucose, methylene blue, 1,1-Dimethylbiguanide hydrochloride (metformin) and exendin-4 were obtained from Sigma-Aldrich, UK. HD-XG glucose telemetry devices were obtained from Data Sciences International, USA.

\section{Results}

Fasting and cage changing cause a biphasic response in blood glucose concentrations

The average $36 \mathrm{~h}$ blood glucose concentrations for both males and females fasted for $6 \mathrm{~h}$ and $16 \mathrm{~h}$ with a bedding retention cage change (BRCC) are shown in Figure 1. This initial plotting of data suggested a biphasic response to fasting prior to a glucose tolerance test (GTT) with 1) initial increases in blood glucose resulting from human intervention to initiate the fast; and 2) prolonged reductions in glucose due to removal of food. Therefore, data was separated and analysed from 0-120 minutes of fast to capture initial responses to intervention and 120-330 minutes (6h fasts) or 120-900 minutes (16h fasts) to capture prolonged responses to fasting.

Blood glucose concentrations initially increase when cages are interfered with for fasting

In both males and females, initiating fasting at $9 \mathrm{am}$ (for $6 \mathrm{~h}$ daytime fasts) with whole cage changes (WCC), bedding retention cage changes (BRCC) and no cage changes (NCC) all produced a transient increase in blood glucose concentration with blood glucose being significantly higher than pre-intervention levels for up to $60 \mathrm{~min}$ in males and $45 \mathrm{~min}$ in females regardless of estrous stage (Figure 2A-C). Initiating fasting at 5pm (for $16 \mathrm{~h}$ overnight fasts) with bedding retention cage change (BRCC) increased blood glucose for up to 45 min in males and $30 \mathrm{~min}$ in females.

The magnitude of the change in blood glucose concentrations was larger in males regardless of cage change method or time of fast initiation with blood glucose increasing by an average of $51.9+-9.1 \%$ in males compared to $23.6+-1.4 \%$ and $27.5+-3.0 \%$ in females in proestrous-estrous (P-E) and metestrous-diestrous (M-D) respectively. This was supported by significantly higher $\mathrm{AUC}_{0-120}$ and difference between maximum and pre-intervention glucose in male mice (Figure 2D-E). Percentage time above pre-intervention glucose was also significantly higher in male mice but only when fasting commenced at 9am (Figure 2E).

Blood glucose concentration increases are most pronounced when the whole cage is changed

The $\mathrm{AUC}_{0-120}$ was highest following whole cage changes in all cohorts regardless of sex or estrous stage (Figure 2D). This appeared to due to a combination of both prolonged responses and increased magnitude of response with whole cage changes tending to cause more pronounced increases in blood glucose and time spent above pre-intervention levels (Figure 2E-F). In all cohorts, 9am fasts produced longer duration of initial responses than $5 \mathrm{pm}$ fasts (Figure $2 \mathrm{~F}$ ).

$6 h$ fasting is least effective when the cage is not changed with $16 \mathrm{~h}$ fasting potentiating blood glucose reductions but to a point of hypoglycaemia

As described above, the initial part of the fast was characterised by increased blood glucose concentrations, most likely caused by researcher intervention and cage changing as more pronounced responses were detected in non-fasted mice (Supplementary figure 1). However, the aim of the fast is to reduce blood glucose concentrations to a basal concentration which should be lower than in the fed scenario. Time taken to reach blood glucose concentrations below fed levels were measured in both male and female fasted mice 
for each cage change condition and fast length. Overall, male mice took longer than female mice to reach blood glucose concentrations below normal fed blood glucose concentrations (Figure 3A-D). In both sexes, the quickest achievement of below-fed-blood glucose concentrations was following 5pm fasting (Figure 3D). Furthermore, for $6 \mathrm{~h}$ fasting starting at 9am, time taken for this effect was quickest when the bedding was retained during the cage change.

To control for normal fluctuations in blood glucose concentrations, fasted mice were compared to mice fed ad libitum. Minimum blood glucose during $6 \mathrm{~h}$ fasts did not fall below normal $24 \mathrm{~h}$ minimum concentrations in the fed state regardless of cage change method (Figure 3E). Conversely, 16h overnight fasting resulted in lower minimum glucose concentrations when compared to both normal $24 \mathrm{~h}$ minimums and $6 \mathrm{~h}$ fasting regardless of cage change method for all cohorts. This minimum occurred at 12.2+-1.2 hours and 14.0+0.7 hours in males and females respectively with some mice becoming hypoglycaemic with blood glucose concentrations less than $2 \mathrm{mM}$.

Under normal ad libitum feeding conditions, blood glucose concentrations only fell below the pre-intervention blood glucose concentrations around 5\% of the time whereas in fasted mice it ranged from between 30-100\%. In all groups, mice fasted for $16 \mathrm{~h}$ spent the highest percentage $(<99 \%)$ of time below pre-intervention levels. In $6 \mathrm{~h}$ fasted mice, not changing the cage resulted in the least time spent below pre-intervention levels particularly in males (Figure $3 \mathrm{~F}$ ).

\section{The cage change method and fast length alters glucose tolerance test outcome}

Intraperitoneal glucose administration increased blood glucose concentration at 15-30 minutes in all mice regardless of cage change method, fast length, sex or estrous. However, 16h fasts were associated with impaired glucose tolerance in all cohorts compared to $6 \mathrm{~h}$ fasts with elevated blood glucose concentrations at 30-60 minutes in males and 30 minutes in females regardless of estrous stage (Figure 4A-C). 6h fasted glucose tolerance was also worsened when the whole cage was changed in males and females in M-D with increased blood glucose at 30 and 15 minutes respectively.

Overall, male mice had impaired glucose tolerance compared to females regardless of estrous stage with higher $\mathrm{AUC}_{120}$ 's. However, this was exaggerated when the whole cage was changed at the start of $6 \mathrm{~h}$ fasting or when mice were fasted overnight (Figure $4 \mathrm{D}$ ). Similarly, $\mathrm{AUC}_{120}$ was significantly higher following whole cage changes and $16 \mathrm{~h}$ fasts in males only.

Oral gels provide further refinement of glucose tolerance test protocol

The experiments described above indicated that 1) 16h fasts with bedding retention cage change (BRCC) cause pronounced hypoglycaemia; 2) $6 \mathrm{~h}$ fasts with a whole cage change (WCC) caused the most pronounced initial increases in blood glucose concentrations; and 3) 6h fasts with no cage change (NCC) were least effective at swiftly reducing blood glucose during the fast. Therefore, $6 \mathrm{~h}$ fasts with bedding retention cage change could be regarded as the most refined protocol whilst ensuring hepatic control of glucose homeostasis (Ayala et al., 2010; Champy et al., 2004; Heijboer et al., 2005; Prior et al., 2012). In an attempt to further refine the protocol by reducing researcher intervention, we compared the effect of voluntary ingestion of gels and oral gavage of glucose. It was found that glucose intake via both oral gels and gavage increased blood glucose at 15-30 minutes in females and 15 minutes in males (Figure 5A-C). However, gavage produced a higher magnitude of blood glucose increase with concentrations at these time-points being significantly higher than gels. Furthermore, the $\mathrm{AUC}_{0-120}$ was significantly higher for gavage vs gels in females regardless of the estrous cycle stage (Figure 5D).

\section{The most refined protocol can still show drug effects}

The experiments above indicated that conditions which involved more severe interventions (whole cage change vs bedding retention, $16 \mathrm{~h}$ fast vs $6 \mathrm{~h}$ fast and oral gavage vs gel) produced the highest blood glucose concentrations during a glucose tolerance test. Therefore, to investigate whether refined procedures were still suitable to detect glucose-lowering effects of clinically relevant drugs, mice were fasted for $6 \mathrm{~h}$ with bedding 
retention cage change before administration of either exendin- 4 or metformin 30 minutes prior to an oral gel glucose tolerance test.

Both metformin and exendin-4 significantly improved glucose tolerance in both males and females with blood glucose being significantly higher for control- vs drug-treated mice at all time-points after glucose administration (Figure $6 \mathrm{~A}-\mathrm{B} ; 5 \mathrm{D}-\mathrm{E}$ ). This was supported by lower $\mathrm{AUC}_{150}$ 's in drug-treated male and female mice (Figure 6C and F).

The difference in means between $\mathrm{AUC}_{150}$ 's and 15 min blood glucose concentration for drug vs controltreated mice tended to be higher in males compared to females (Table 2). However, the standard deviation for these variables also tended to be higher in males. Consequently, sample size calculations with a desired power of 0.90 and alpha of 0.01 revealed that the same number of male and female mice would be required to detect a statistical difference between exendin or metformin vs controls.

\section{Discussion and Conclusions}

Despite being one of the most commonly used tools in diabetes research, there is no standardisation of the glucose tolerance test (GTT) procedure. Overall, our data shows that $6 \mathrm{~h}$ fasts with bedding retention cage changes (BRCC) followed by oral glucose gels at the start of the GTT, supported protocol refinement whilst retaining the ability to demonstrate metformin and exendin efficacy in both males and females. Importantly, our results have also shown that even minor variations in GTT protocol, which may seem inconsequential to researchers, can affect outcome. Moreover, we have generated data that show that in the most refined protocols, the estrous stage does not affect results. Indeed, irrespective of estrous stage, female mice showed reduced blood glucose variability during GTTs compared to male mice. Consequently, we used two clinically relevant drugs to challenge a perception that a milder response to a glucose challenge in females would impede their ability to demonstrate the effects of anti-diabetic drugs.

Lack of standardisation between different studies and consequent deviations in protocols, could be problematic given that severe interventions, such as whole cage changing and overnight fasting, are associated with stress responses (Ayala et al., 2010; Jensen et al., 2013; Rasmussen et al., 2011). This could affect the interand intra- laboratory reproducibility of data due to the impact of stress on blood glucose concentrations, and negatively impact research and development, where therapeutic entities developed for diabetes treatment are routinely assayed using GTTs. Furthermore, there may be welfare implications of more severe interventions that should be minimised where possible in line with the 3Rs.

Indeed, it is essential that researchers have a solid understanding of how their own interventions and study design may impact both welfare and experimental outcomes. This has previously been difficult to determine due to the need for tail vein blood sampling using standard glucometer methods, which may in itself unintentionally alter blood glucose concentrations due to researcher intervention. However, telemetric glucose monitoring now provides a means for continuous measurement of blood glucose concentrations without necessitating researcher intervention. Therefore, this study used continuous glucose monitoring to analyse the impact of pre-GTT interventions on blood glucose and GTT outcome and to determine whether these effects can be minimised with refined procedures.

Further potential issues with regards to translatability of preclinical research is the common exclusion of female mice. This is often due to a combination of perceived increased variability in physiological parameters due to the estrous cycle, and milder diabetic phenotype compared to males (Bartke et al., 1973; Beery, 2018; Kaikaew et al., 2019; Pettersson et al., 2012) . The assumption of high variability in other scientific disciplines has largely been addressed with research showing higher variability in male mice (Beery, 2018).

Although it is widely accepted that females become less hyperglycaemic, glucose intolerant and insulin resistant in almost all models of diabetes, their exclusion from preclinical studies may not be justified. Even in normoglycaemic mice, differences in male and female glucose tolerance have been described (Bowe et al., 2014; Goren et al., 2004; Macotela et al., 2009) and our study confirms this sex difference. However, this modest phenotype in female mice does not necessarily justify their exclusion from preclinical studies. 
Indeed, with significant evidence of sex differences in human diabetes and response to several anti-diabetic agents, the inclusion of females in the preclinical setting may be particularly pertinent (Arnetz et al., 2014; Kautzky-Willer et al., 2016). Furthermore, there is now increasing emphasis on the need to better consider sex as a biological variable across all scientific disciplines (Beery, 2018; Clayton \& Collins, 2014; Docherty et al., 2019). Therefore, understanding the impact of sex on outcomes is vital to determine the suitability of female mice, especially when attempting to detect drug effects because of their reduced diabetic phenotype.

By reducing the need to move animals out of the holding room for experimentation, we have been able to establish the physiological changes associated specifically with cage changing, fasting duration and sex independently of stress associated with transport of animals and/or a novel room. Our results show a biphasic response to fasting and cage changing with an initial increase in blood glucose associated with human intervention of the cage change followed by a reduction in glucose due to fasting. Different pre-GTT interventions can impact the effect of fasting with whole cage changes being associated with exaggerated initial blood glucose increases at the start of a $6 \mathrm{~h}$ daytime fast. Furthermore, male mice exhibited increased initial responses (higher magnitude and duration of blood glucose increases) to intervention than females regardless of cage change method, fast length or estrous stage.

Prolonged effects of fasting also differed depending on cage change method and fast length with $6 \mathrm{~h}$ fasting without changing the cage resulting in more variable, less pronounced and less timely glucose reductions. Overnight fasting was associated with pronounced hypoglycaemia with blood glucose consistently falling below $2 \mathrm{mM}$ at $\sim 13 \mathrm{~h}$ after the start of the fast. Although evidence of overnight-fasted hypoglycaemia has previously been documented, we have been able to show exactly how severe the hypoglycaemia can become (Jensen et al., 2013). Previous studies using discontinuous glucometer readings miss this, firstly because the minimum blood glucose tends to be reached about $3 \mathrm{~h}$ before the fast ends, which is not at a timepoint the researchers typically measure. Secondly, handling the animal acutely increases blood glucose concentrations which masks the extent of the blood glucose concentrations nadir reached during the fast. Indeed, remote telemetric data revealed that animals had reached a profoundly hypoglycaemic nadir of $2 \mathrm{mM}$ when undisturbed, but the conventional process of handling the animal at the $16 \mathrm{~h}$ time point, for a pre-GTT baseline glucometer reading, doubled this to $\sim 4 \mathrm{mM}$. It is worth noting that evidence of hypothermia and significant weight loss was also observed (Supplementary Figure 2). Conversely, $6 \mathrm{~h}$ fasting was not associated with the same welfare concerns regardless of cage change method, sex or estrous stage.

Although more studies are now using shorter fasts, a review of the literature using the key phrase 'glucose tolerance test and mouse' indicated that $60 \%$ of studies are still fasting mice overnight. Our results not only show that this has welfare implications but that it induces glucose intolerance in male mice. Indeed, differing pre-GTT methods can have profound effects on GTT outcomes. Overall, males were more glucose intolerant than females regardless of cage change method, fast length or estrous stage. However, these differences were exaggerated when mice were fasted overnight or when the whole cage was changed at the start of a $6 \mathrm{~h}$ daytime fast. Indeed, overnight fasting and whole cage changes caused impaired glucose tolerance particularly in male mice. The estrous cycle had no significant impact on outcomes although females in metestrous-diestrous (MD) tended to be more glucose intolerant than those in proestrous-estrous (P-E) particularly when more severe interventions (16h fasting and whole cage changes) were undertaken. This supports previous research and is likely to be due to lower concentrations of estrogen during M-D although this was not directly measured (Mauvais-Jarvis, 2015). Indeed, estrogen is known to improve glucose tolerance and reduce sensitivity to stressors, both of which are likely to have played a role in our outcomes (Lovick, 2012). Overall, these results show how the severity of pre-GTT intervention can alter scientific outcomes and potentially affect interpretation of the degree of glucose tolerance. Furthermore, our data suggests that estrous cycling is unlikely to alter outcomes if procedures are refined in mice.

Finally, our data showed that further refinement via the use of voluntary oral glucose gels can still provide reproducible blood glucose increases during glucose tolerance tests. However, administration of glucose by the mice voluntarily ingesting glucose gels led to lower glucose peaks post administration compared to oral gavage. Although we cannot rule out the possibility of enhanced incretin effects with glucose gels due to 
chewing, the differences observed are also likely to be associated with the reduced stress of voluntary gel consumption (Fujiwara et al., 2019).

Overall, glucose tolerance was improved in female mice and was improved by refinement of procedures (6h vs $16 \mathrm{~h}$ fasts, bedding retention vs whole cage change, gels vs gavage). Refinement is important in animal welfare and the use of females is important to ensure that the heterogeneous nature of blood glucose homeostasis is captured. However, the relatively mild changes seen during the GTT following these procedures may preclude their suitability when studying drug effects which would impede on the predictive validity of the model. Therefore, our final experiment aimed to use the most refined procedures to determine whether higher blood glucose increases, whether due to more severe intervention, route of glucose administration or sex, is required for reliable drug efficacy studies. It was found that the effect of both metformin and exendin-4 could be observed using oral glucose gels in both males and females. Furthermore, although magnitude of drug effect was attenuated in female mice, the inter-mouse variability of their responses was also reduced. Consequently, sample size calculations revealed that the same number of male and female mice would be required to detect the effects of both drugs.

This study highlights the potential impact of differing pre-GTT interventions on outcomes and shows that refinement of procedures can maximise welfare in line with the 3Rs. Consequently, the results emphasise the impact of experimental design on outcomes and hence the value of GTT standardisation and full disclosure of all steps of the GTT protocol from the point of fasting. Furthermore, the importance of using females and considering sex as a variable has been highlighted with clear biological sex differences with regards to response to intervention and glucose tolerance. Although the mice studied were normoglycaemic, this research provides proof-of-concept to question the commonly held belief that higher blood glucose concentrations are required to see the effect of anti-diabetic drugs. Indeed, we have shown that despite improved glucose tolerance associated with refinement of procedures and the use of female mice, the ability to detect drug effects during glucose tolerance tests is not affected. The research also refutes other concerns about the inclusion of females in preclinical studies including perceived increased variability, the need to consider estrous cycling, and the requirement for higher sample sizes. In conclusion, this study highlights the importance of experimental design and inclusion of females in studies of blood glucose homeostasis and provides evidence to refute the perception that more severe phenotypes are required for studying drug effects.

Figure 1. $36 \mathrm{~h}$ average 10 second blood glucose concentrations following $6 \mathrm{~h}$ fast (commencing at 9am) or $16 \mathrm{~h}$ fast (commencing at $5 \mathrm{pm}$ ) with bedding retention cage changes (BRCC) and subsequent intraperitoneal glucose tolerance tests (GTTs) for A) Males andB) Females. Black line = blood glucose concentrations following $6 \mathrm{~h}$ fast with BRCC; red line = blood glucose concentrations following $16 \mathrm{~h}$ fast with BRCC. Grey bar $=$ dark phase. Data are mean+-SEM ( $\mathrm{n}=7$ for males and females).

Figure 2. The initial effects of $6 \mathrm{~h}$ and $16 \mathrm{~h}$ fasting on blood glucose concentrations from -30 minutes preintervention to 120 minutes post-intervention in A) Males; B) Females in proestrous-estrous (P-E); and C) Females in metestrous-diestrous (M-D). D) $\mathrm{AUC}_{120}$ for graphs A-C. E) Difference between maximum blood glucose reached from 0-120 minutes following intervention and pre-intervention baseline.F) Percentage time spent above pre-intervention blood glucose concentrations from 0-120 minutes post-intervention. Preintervention glucose was quantified as the average glucose between -30 and -15 minutes. $\mathrm{WCC}=$ whole cage change; $\mathrm{BRCC}=$ bedding retention cage change; $\mathrm{NCC}=$ no cage change; $\mathrm{P}-\mathrm{E}=$ proestrous-estrous; $\mathrm{M}-\mathrm{D}$ $=$ metestrous-diestrous. ${ }^{*}$ and \# represent a significant difference compared to females in P-E and M-D respectively. $\sim,+,=$ and ? represent a significant difference compared to $6 \mathrm{~h}$ with WCC, $6 \mathrm{~h}$ with BRCC, $6 \mathrm{~h}$ with NCC and $16 \mathrm{~h}$ with BRCC respectively ( $\mathrm{p}<0.05$, two-way ANOVA with Holm-Sidak post-hoc tests). Data are mean+-SEM ( $n=7$ for males, $n=5-7$ for females in P-E and $n=4-7$ for females in M-D).

Figure 3. The prolonged effects of $6 \mathrm{~h}$ and $16 \mathrm{~h}$ fasting on blood glucose concentrations from - 30 minutes preintervention to 330 minutes and 960 minutes post-intervention for $6 \mathrm{~h}$ and $16 \mathrm{~h}$ fasts respectively inA) Males; B) Females in proestrous-estrous (P-E); andC) Females in metestrous-diestrous (M-D). D) Time after start of fast that blood glucose fell below pre-intervention concentrations for 5 consecutive minutes. E) Minimum blood glucose concentration reached during fasting compared to normal $24 \mathrm{~h}$ minimum glucose concentrations 
with ad libitum food. F)Percentage time spent below pre-intervention blood glucose concentrations from 0330 or 0-960 minutes post-intervention compared to normal ad libitum conditions. Pre-intervention glucose was quantified as the average glucose between -30 and -15 minutes. $\mathrm{WCC}=$ whole cage change; $\mathrm{BRCC}$ $=$ bedding retention cage change; $\mathrm{NCC}=$ no cage change; $\mathrm{P}-\mathrm{E}=$ proestrous-estrous; $\mathrm{M}-\mathrm{D}=$ metestrousdiestrous. * and \# represent a significant difference compared to females in P-E and M-D respectively. , +, $=, \mathrm{Y}=$ and ? represent a significant difference compared to $6 \mathrm{~h}$ with WCC, $6 \mathrm{~h}$ with BRCC, $6 \mathrm{~h}$ with NCC, ad libitum conditions and $16 \mathrm{~h}$ with BRCC respectively $(\mathrm{p}<0.05$, two-way ANOVA with Holm-Sidak post-hoc tests). Data are mean+-SEM ( $n=7$ for males, $n=5-7$ for females in P-E and $n=4-7$ for females in M-D).

Figure 4. The effect of $6 \mathrm{~h}$ and $16 \mathrm{~h}$ fasts with different cage change methods on intraperitoneal glucose tolerance test (IPGTT) outcome in A) Males; B) Females in proestrous-estrous (P-E); and C) Females in metestrous-diestrous (M-D). D) $\mathrm{AUC}_{120}$ for graphs $\mathrm{A}-\mathrm{C}$. $\mathrm{WCC}=$ whole cage change; $\mathrm{BRCC}=$ bedding retention cage change; $\mathrm{NCC}=$ no cage change; $\mathrm{P}-\mathrm{E}=$ proestrous-estrous; $\mathrm{M}-\mathrm{D}=$ metestrous-diestrous. ${ }^{*}$ and \# represent a significant difference compared to females in P-E and M-D respectively. $~ \sim,+$ and = represent a significant difference compared to $6 \mathrm{~h}$ with WCC, $6 \mathrm{~h}$ with BRCC and $6 \mathrm{~h}$ with NCC respectively ( $\mathrm{p}<0.05$, two-way RM ANOVA with Holm-Sidak post-hoc tests). Data are mean+-SEM ( $\mathrm{n}=7$ for males, females in P-E and females in M-D).

Figure 5. The effect of oral gavage and oral glucose gels on $6 \mathrm{~h}$ fasted intraperitoneal glucose tolerance test (IPGTT) outcome following bedding retention cage changes (BRCC) in A) Males; B)Females in proestrousestrous (P-E); and C) Females in metestrous-diestrous (M-D). D) $\mathrm{AUC}_{120}$ for graphs A-C. P-E = proestrousestrous; $\mathrm{M}-\mathrm{D}=$ metestrous-diestrous. ${ }^{*}$ and \# represent a significant difference compared to females in P-E and M-D respectively. represents a significant difference compared to oral glucose gels $(\mathrm{p}<0.05$, two-way RM ANOVA with Holm-Sidak post-hoc tests). Data are mean+-SEM ( $\mathrm{n}=5$ for males, females in P-E and females in M-D).

Figure 6. The effect of oral metformin and intraperitoneal exendin- 4 on $6 \mathrm{~h}$ fasted intraperitoneal glucose tolerance test (IPGTT) outcome following bedding retention cage changes (BRCC). A-B)I.p. exendin-4 vs i.p. saline control in A) Males andB) Females. D-E) Oral metformin vs sweetener gel control in D) Males and E) Females. $\mathbf{C}$ and $\mathbf{F}) \mathrm{AUC}_{120}$ for graphs A-B and D-E respectively. ${ }^{*}$ and \# represent a significant difference compared to females and control respectively $(\mathrm{p}<0.05$, two-way ANOVA with Holm-Sidak post-hoc tests). Data are mean+-SEM ( $\mathrm{n}=4$ for males and females).

Table 1. Cage changing methods used at the start of $6 \mathrm{~h}$ daytime and $16 \mathrm{~h}$ overnight fasting. Ticks and crosses represent whether or not this occurred for that particular method.

Table 2. Sample size calculations for $250 \mathrm{mg} / \mathrm{kg}$ oral metformin and $10 \mathrm{nmol} / \mathrm{kg}$ i.p. exendin- 4 versus control glucose tolerance tests (GTTs). Calculations were undertaken using the difference in means and standard deviations for the $\mathrm{AUC}_{150}$ for metformin vs sweetener control and exendin- 4 vs saline control and 15 minute post-glucose glucometer blood glucose concentration in both males and females.

Supplementary Figure 1. The effect of husbandry whole cage changes without fasting on blood glucose concentrations from -30 minutes pre-intervention to 150 minutes post-intervention in males and females(A) . ${ }^{*}$ and \# represent a significant difference compared to females and baseline respectively $(\mathrm{p}<0.05$, twoway ANOVA with Holm-Sidak post-hoc tests). Black bar: different between males and females. Blue bar: males higher than baseline. Red bar: females higher than baseline. Data are mean+-SEM ( $\mathrm{n}=7$ males and females). B) $\mathrm{AUC}_{0-150}$ for graph A. C) Difference between maximum blood glucose reached from 0-150 minutes following intervention and pre-intervention baseline. D) Percentage time spent above preintervention blood glucose concentrations from 0-150 minutes post-intervention. Pre-intervention glucose was quantified as the average glucose between -30 and -15 minutes. * represents a significant difference compared to females ( $\mathrm{p}<0.05$, t-test). Data are mean+-SEM ( $\mathrm{n}=7$ for males and females).

Supplementary Figure 2. The effect of $16 \mathrm{~h}$ fasting on body temperature from -30 minutes pre-intervention to 900 minutes post-intervention in males and females (A) . B) Minimum body temperature reached during $6 \mathrm{~h}$ and $16 \mathrm{~h}$ fasting compared to normal $24 \mathrm{~h}$ minimum temperatures with ad libitum food. C) Percentage 
weight loss in the $24 \mathrm{~h}$ whereby a $6 \mathrm{~h}$ and $16 \mathrm{~h}$ fast was undertaken. * represents a significant difference compared to females and ? represents a significant difference compared to $16 \mathrm{~h}$ fasts $(\mathrm{p}<0.05$, two-way RM ANOVA with Holm-Sidak post-hoc tests). Data are mean+-SEM ( $\mathrm{n}=7$ for males and females).

\section{References}

Andrikopoulos, S., Blair, A. R., Deluca, N., Fam, B. C., \& Proietto, J. (2008). Evaluating the glucose tolerance test in mice. American Journal of Physiology-Endocrinology and Metabolism , 295 (6), E1323E1332. https://doi.org/10.1152/ajpendo.90617.2008

Arnetz, L., Ekberg, N. R., \& Alvarsson, M. (2014). Sex differences in type 2 diabetes: Focus on disease course and outcomes. Diabetes, Metabolic Syndrome and Obesity: Targets and Therapy , 7, 409-420. https://doi.org/10.2147/DMSO.S51301

Ayala, J. E., Samuel, V. T., Morton, G. J., Obici, S., Croniger, C. M., Shulman, G. I., Wasserman, D. H., \& McGuinness, O. P. (2010). Standard operating procedures for describing and performing metabolic tests of glucose homeostasis in mice. Disease Models 83 Mechanisms ,3 (9-10), 525-534. https://doi.org/10.1242/dmm.006239

Bartke, A., Steele, R. E., Musto, N., \& Caldwell, B. V. (1973). Fluctuations in Plasma Testosterone Levels in Adult Male Rats and Mice.Endocrinology , 92 (4), 1223-1228. https://doi.org/10.1210/endo-92-4-1223

Beery, A. K. (2018). Inclusion of females does not increase variability in rodent research studies. Current Opinion in Behavioral Sciences , 23 , 143-149. https://doi.org/10.1016/j.cobeha.2018.06.016

Bowe, J. E., Franklin, Z. J., Hauge-Evans, A. C., King, A. J., Persaud, S. J., \& Jones, P. M. (2014). METABOLIC PHENOTYPING GUIDELINES: Assessing glucose homeostasis in rodent models. Journal of Endocrinology , 222 (3), G13-G25. https://doi.org/10.1530/JOE-14-0182

Byers, S. L., Wiles, M. V., Dunn, S. L., \& Taft, R. A. (2012). Mouse Estrous Cycle Identification Tool and Images. PLOS ONE ,7 (4), e35538. https://doi.org/10.1371/journal.pone.0035538

Champy, M.-F., Selloum, M., Piard, L., Zeitler, V., Caradec, C., Chambon, P., \& Auwerx, J. (2004). Mouse functional genomics requires standardization of mouse handling and housing conditions.Mammalian Genome: Official Journal of the International Mammalian Genome Society , 15 (10), 768-783. https://doi.org/10.1007/s00335-004-2393-1

Clayton, J. A., \& Collins, F. S. (2014). Policy: NIH to balance sex in cell and animal studies. Nature News , 509 (7500), 282. https://doi.org/10.1038/509282a

Cox, H. M., Tough, I. R., Woolston, A.-M., Zhang, L., Nguyen, A. D., Sainsbury, A., \& Herzog, H. (2010). Peptide YY is critical for acylethanolamine receptor Gpr119-induced activation of gastrointestinal mucosal responses. Cell Metabolism, 11 (6), 532-542. https://doi.org/10.1016/j.cmet.2010.04.014

Curtis, M. J., Alexander, S., Cirino, G., Docherty, J. R., George, C. H., Giembycz, M. A., Hoyer, D., Insel, P. A., Izzo, A. A., Ji, Y., MacEwan, D. J., Sobey, C. G., Stanford, S. C., Teixeira, M. M., Wonnacott, S., \& Ahluwalia, A. (2018). Experimental design and analysis and their reporting II: Updated and simplified guidance for authors and peer reviewers. British Journal of Pharmacology , 175 (7), 987-993. https://doi.org/10.1111/bph.14153

Dhawan, S. S., Xia, S., Tait, D. S., Bundgaard, C., Bowman, E., \& Brown, V. J. (2018). Oral dosing of rodents using a palatable tablet.Psychopharmacology , 235 (5), 1527-1532. https://doi.org/10.1007/s00213018-4863-2

Docherty, J. R., Stanford, S. C., Panettieri, R. A., Alexander, S. P. H., Cirino, G., George, C. H., Hoyer, D., Izzo, A. A., Ji, Y., Lilley, E., Sobey, C. G., Stanley, P., Stefanska, B., Stephens, G., Teixeira, M., \& Ahluwalia, A. (2019). Sex: A change in our guidelines to authors to ensure that this is no longer an ignored experimental variable.British Journal of Pharmacology , 176 (21), 4081-4086. https://doi.org/10.1111/bph.14761 
Fujiwara, Y., Eguchi, S., Murayama, H., Takahashi, Y., Toda, M., Imai, K., \& Tsuda, K. (2019). Relationship between diet/exercise and pharmacotherapy to enhance the GLP-1 levels in type 2 diabetes.Endocrinology, Diabetes $\&$ Metabolism, 2 (3), e00068. https://doi.org/10.1002/edm2.68

Goren, H. J., Kulkarni, R. N., \& Kahn, C. R. (2004). Glucose Homeostasis and Tissue Transcript Content of Insulin Signaling Intermediates in Four Inbred Strains of Mice: C57BL/6, C57BLKS/6, DBA/2, and 129X1. Endocrinology , 145 (7), 3307-3323. https://doi.org/10.1210/en.2003-1400

Heijboer, A. C., Donga, E., Voshol, P. J., Dang, Z.-C., Havekes, L. M., Romijn, J. A., \& Corssmit, E. P. M. (2005). Sixteen hours of fasting differentially affects hepatic and muscle insulin sensitivity in mice.Journal of Lipid Research , 46 (3), 582-588. https://doi.org/10.1194/jlr.M400440-JLR200

Jensen, T., Kiersgaard, M., Sorensen, D., \& Mikkelsen, L. (2013). Fasting of mice: A review. Laboratory Animals , 47 (4), 225-240. https://doi.org/10.1177/0023677213501659

Jensen, V. S., Porsgaard, T., Lykkesfeldt, J., \& Hvid, H. (2016). Rodent model choice has major impact on variability of standard preclinical readouts associated with diabetes and obesity research.American Journal of Translational Research , 8 (8), 3574-3584.

Kaikaew, K., Steenbergen, J., van Dijk, T. H., Grefhorst, A., \& Visser, J. A. (2019). Sex Difference in Corticosterone-Induced Insulin Resistance in Mice. Endocrinology , 160 (10), 2367-2387. https://doi.org/10.1210/en.2019-00194

Kautzky-Willer, A., Harreiter, J., \& Pacini, G. (2016). Sex and Gender Differences in Risk, Pathophysiology and Complications of Type 2 Diabetes Mellitus. Endocrine Reviews , 37 (3), 278-316. https://doi.org/10.1210/er.2015-1137

King, A. J. F. (2012). The use of animal models in diabetes research.British Journal of Pharmacology , 166 (3), 877-894. https://doi.org/10.1111/j.1476-5381.2012.01911.x

Lovick, T. A. (2012). Estrous cycle and stress: Influence of progesterone on the female brain. Brazilian Journal of Medical and Biological Research , 45 (4), 314-320. https://doi.org/10.1590/S0100-879X2012007500044

Macotela, Y., Boucher, J., Tran, T. T., \& Kahn, C. R. (2009). Sex and depot differences in adipocyte insulin sensitivity and glucose metabolism. Diabetes , 58 (4), 803-812. https://doi.org/10.2337/db08-1054

Mauvais-Jarvis, F. (2015). Sex differences in metabolic homeostasis, diabetes, and obesity. Biology of Sex Differences , 6 (1), 14. https://doi.org/10.1186/s13293-015-0033-y

McGuinness, O. P., Ayala, J. E., Laughlin, M. R., \& Wasserman, D. H. (2009). NIH experiment in centralized mouse phenotyping: The Vanderbilt experience and recommendations for evaluating glucose homeostasis in the mouse. American Journal of Physiology-Endocrinology and Metabolism, 297 (4), E849-E855. https://doi.org/10.1152/ajpendo.90996.2008

Pacini, G., Omar, B., \& Ahren, B. (2013, May 21). Methods and Models for Metabolic Assessment in Mice [Methodology Report]. Journal of Diabetes Research. https://doi.org/10.1155/2013/986906

Pedro, P. F., Tsakmaki, A., \& Bewick, G. A. (2020). The Glucose Tolerance Test in Mice. Methods in Molecular Biology (Clifton, N.J.) , 2128 , 207-216. https://doi.org/10.1007/978-1-0716-0385-7_14

Pettersson, U. S., Walden, T. B., Carlsson, P.-O., Jansson, L., \& Phillipson, M. (2012). Female Mice are Protected against High-Fat Diet Induced Metabolic Syndrome and Increase the Regulatory T Cell Population in Adipose Tissue. PLoS ONE , 7 (9). https://doi.org/10.1371/journal.pone.0046057

Prior, H., Ewart, L., Bright, J., \& Valentin, J.-P. (2012). Refinement of the charcoal meal study by reduction of the fasting period.Alternatives to Laboratory Animals: ATLA , 40 (2), 99-107. https://doi.org/10.1177/026119291204000209 
Rasmussen, S., Miller, M. M., Filipski, S. B., \& Tolwani, R. J. (2011). Cage Change Influences Serum Corticosterone and Anxiety-Like Behaviors in the Mouse. Journal of the American Association for Laboratory Animal Science : JAALAS , 50 (4), 479-483.

Seino, Y., \& Yabe, D. (2013). Glucose-dependent insulinotropic polypeptide and glucagon-like peptide1: Incretin actions beyond the pancreas. Journal of Diabetes Investigation , 4 (2), 108-130. https://doi.org/10.1111/jdi.12065

Turner, P. V., Brabb, T., Pekow, C., \& Vasbinder, M. A. (2011). Administration of Substances to Laboratory Animals: Routes of Administration and Factors to Consider. Journal of the American Association for Laboratory Animal Science : JAALAS , 50 (5), 600-613.

Zhang, L. (2011). Voluntary oral administration of drugs in mice.Protocol Exchange . https://doi.org/10.1038/protex.2011.236

Zhang, L. (2021). Method for voluntary oral administration of drugs in mice. STAR Protocols , 2 (1), 100330. https://doi.org/10.1016/j.xpro.2021.100330

\section{Hosted file}

Figure 1.docx available at https://authorea.com/users/423117/articles/528663-the-glucosetolerance-test-in-mice-sex-drugs-and-protocol

\section{Hosted file}

Figure 2.docx available at https://authorea.com/users/423117/articles/528663-the-glucosetolerance-test-in-mice-sex-drugs-and-protocol

\section{Hosted file}

Figure 3.docx available at https://authorea.com/users/423117/articles/528663-the-glucosetolerance-test-in-mice-sex-drugs-and-protocol

\section{Hosted file}

Figure 4.docx available at https://authorea.com/users/423117/articles/528663-the-glucosetolerance-test-in-mice-sex-drugs-and-protocol

\section{Hosted file}

Figure 5.docx available at https://authorea.com/users/423117/articles/528663-the-glucosetolerance-test-in-mice-sex-drugs-and-protocol

\section{Hosted file}

Figure 6.docx available at https://authorea.com/users/423117/articles/528663-the-glucosetolerance-test-in-mice-sex-drugs-and-protocol

\section{Hosted file}

Table 1.docx available at https://authorea.com/users/423117/articles/528663-the-glucosetolerance-test-in-mice-sex-drugs-and-protocol

\section{Hosted file}

Table 2.docx available at https://authorea.com/users/423117/articles/528663-the-glucosetolerance-test-in-mice-sex-drugs-and-protocol

\section{Hosted file}

Supplementary Figure 1.docx available at https://authorea.com/users/423117/articles/528663the-glucose-tolerance-test-in-mice-sex-drugs-and-protocol 


\section{Hosted file}

Supplementary Figure 2.docx available at https://authorea.com/users/423117/articles/528663the-glucose-tolerance-test-in-mice-sex-drugs-and-protocol 